\title{
Article
}

\section{Topical Triamcinolone Acetonide-Loaded Liposomes Formula- tion Used as an Adjuvant to Intravitreal Ranibizumab Therapy for Neovascular Age Related Macular Degeneration}

Jose Navarro-Partida ${ }^{1,2^{*}}$, Juan Carlos Altamirano-Vallejo ${ }^{1,2^{*}}$, Luis Abraham Aceves Franco ${ }^{1,2}$, Jesús Gonzalez-Cortes ${ }^{3}$, Sergio Hernandez Da Mota ${ }^{4}$, Jose Gerardo García-Aguirre1,5, Carlos David Azuara-Galindo6, Carlos Rodrigo Castro-Castaneda ${ }^{1}$, Juan Armendariz-Borunda ${ }^{1,7}$, Arturo Santos ${ }^{1,2+}$

1 Tecnologico de Monterrey, Escuela de Medicina y Ciencias de la Salud, Monterrey, N.L., México

2 Centro de Retina Medica y Quirúrgica, S.C., Hospital Puerta de Hierro. Zapopan, Jalisco, México Universidad Autonoma de Nuevo Leon, Hospital “Dr. Jose Eleuterio Gonzalez”, Facultad de Medicina, Monterrey, Nuevo Leon, Mexico

4 Clinica David. Unidad Oftalmologia. Servicio de Retina, Morelia, Michoacan, Mexico

5 Asociacion para Evitar la Ceguera en Mexico I.A.P., Ciudad de Mexico, Mexico.

6 ISSSTE Clinica Hospital Constitucion Monterrey, Nuevo Leon, Mexico

Department of Molecular Biology and Genomics, Institute for Molecular Biology and Gene Therapy, University of Guadalajara, Sierra Mojada 950, Colonia Independencia, Edificio Q. Tercer Piso, CP 44340 Guadalajara, Jalisco, Mexico

+ Correspondence: arturo.santos@tec.mx, asantos@e-retina.com; Tel.: +52 (33) 36693000 ext: 2540

* These authors contributed equally to this work.

Abstract: Novel strategies have been developed to reduce or to avoid the intravitreal injections (IVTs) of the antiangiogenic (ranibizumab; RBZ) and the anti-inflammatory (triamcinolone acetonide; TA) agents used to treat vitreoretinal diseases. One of the strategies include liposomes. In this study, it was evaluated the safety and efficacy of topical triamcinolone-loaded liposomes formulation (TALF) as an adjuvant to intravitreal RBZ therapy in treatment-naive patients with neovascular age related macular degeneration (nAMD). Subjects were randomly assigned to the RBZ TALF or the RBZ-pro re nata (RBZ-PRN) group. Patients from the RBZ-TALF group were instructed to apply TALF for a 12-month period after a single dose of RBZ. Patients from the RBZ-PRN group received three monthly RBZ-IVTs. Retreatment with RBZ was considered in case of nAMD reactivation. Related to safety, non-ocular abnormalities were observed during TALF therapy. Owing to the efficacy, non-significant differences are identified in visual acuity or central foveal thickness when the RBZ-PRN and RBZ-TALF groups are compared. Importantly the average number of RBZ injections was significantly lower in the RBZ-TALF group ( $2.5 \pm 1.4$ vs $6.1 \pm 1.3$ IVTs; $p=0004)$. Therefore, TALF used as an adjuvant to RBZ reduce the number of RBZ-IVTs retreatment with optimal visual and anatomic results.

Keywords: Triamcinolone acetonide; Liposomes; Neovascular age related macular degeneration Wet macular degeneration; Adjuvant therapy; Ranibizumab

\section{Introduction}

Age related macular degeneration (AMD) has been targeted as the main cause of irreversible blindness in over 50 years and older population in developed countries [1-4]. Worldwide, around 17 million people are living with this condition [3, 5]. AMD can be classified into neovascular (wet) and non-neovascular (dry) form. Although the neovascular form represents only $10 \%-20 \%$ of all cases of AMD, $80-90 \%$ of severe visual loss cases are explained by it. Vision impairment in neovascular AMD (nAMD) follows the development of choroidal neovascularization in the macula. These vascular changes increase the fluid leakage with the consequent thickening and late stage scarring of the central retina leading to important central vision loss [6]. 
Vascular endothelial growth factor (VEGF) has been demonstrated to be the most important angiogenic factor responsible for choroidal neovascularization (CNV) in nAMD. However, several lines of evidence implicate the immune system and different inflammatory pathways in the nAMD pathogenesis. Chronic inflammatory cells such as fibroblasts, macrophages and circulating progenitor/stem cells have been reported in surgically excised subfoveal neovascular membranes, as well as the accumulation of collagen, basal laminar deposits and fibrin which comprise the extracellular components [7-12]. It has also been described that IL-1- $\beta$ and TNF- $\alpha$ secreted by macrophages may promote choroidal neovascularization [13]. Therefore, CNV is a process which involves inflammation and neovascularization. Owing to the participation of angiogenic mechanisms in the nAMD pathogenesis, molecules targeting angiogenesis (anti-VEGF agents) such as ranibizumab (RBZ), aflibercept and the off-label bevacizumab have been used intravitreally for over a decade to treat patients with nAMD, slowing disease progression and preserving and improving vision [14-17]. In fact, intravitreal anti-VEGF therapy is currently the standard of care in patients with nAMD [18].

Even though the benefits of intravitreal injections (IVTs) of anti-VEGF drugs in nAMD has been well documented, this procedure is linked to potential complications resulting from the route of administration, such as endophthalmitis, retinal detachment and traumatic cataract $[19,20]$. Moreover, large bolus of anti-VEGF agents in long-term treatment has been related to macular atrophy [21, 22].

In the last years, novel therapeutic strategies to reduce or to avoid the use of intravitreal injections have been developed. These strategies include the improvement of treatment interval [23], sustained drug delivery system [24, 25], implantable drug delivery systems [26-28] and novel drug carriers systems such as, microemulsions, nanosuspensions, nanoparticles and liposomes [29-32]. One of these promising approaches are the liposome based nanocarriers. Liposomes are biocompatible vesicles which share structural characteristics, including a phospholipid-bilayer, with the cell membrane and carry both hydrophilic and lipophilic drugs in their small specific spheroids shape [33, 34].

Recently, a triamcinolone acetonide-loaded liposome formulation (TALF) was designed to be used topically to release triamcinolone acetonide (TA), a well-known synthetic corticosteroid, into the vitreous cavity and to reach the retinal tissue. The safety, tolerability and efficacy of this novel liposomal formulation was successfully tested in rabbits [35], while its therapeutic activity was clearly verified in patients with refractory pseudophakic cystoid macular edema, diabetic macular edema and macular edema secondary to branch retinal vein occlusion [36-38]. TALF demonstrated to enable continuous, controlled, and effective release of triamcinolone acetonide into the posterior segment tissues and was also well tolerated without presenting adverse events such as ocular hypertension or cataract progression $[36,38]$.

Since inflammatory and angiogenic pathways contribute to the development of neovascularization by increasing the vascular permeability in the wet form of AMD, a therapy which suppresses both pathways has been postulated to produce sustainable effects and may have advantages compared to the mono-therapeutic approaches [39, 40]. Indeed, combination therapies with anti-VEGF agents and TA, have shown positive effects in the therapy of nAMD, and have become increasingly common in recent years [41-44]. Due to the highly complex process the pathogenesis of $\mathrm{CNV}$ represents, where both angiogenesis and inflammation play an important role, it is hypothesized that using the topical ophthalmic TALF as an adjuvant to the intravitreal injection of RBZ, could enhance efficacy of the intravitreal anti-VEGF agent and minimize unwanted collateral effects by reducing the doses and the number of intravitreal injections already used in the monotherapy with RBZ. Therefore, in this clinical assay, we explored and reported the feasibility and safety 
of using topical TALF as an adjuvant to intravitreal anti-VEGF therapy with ranibizumab for nAMD.

\section{Methods}

\subsection{Study Design}

To evaluate feasibility and safety of topical TALF used as an adjuvant to intravitreal anti-VEGF therapy with RBZ for nAMD, a single-center Phase II clinical trial was conducted in patients with diagnosis of nAMD at a private-based, ISO 9001:20015 Certified research unit in ophthalmology in Guadalajara, Mexico (Centro de Retina Medica y Quirurgica, SC). An external review board approval authorization was obtained before enrollment of patients (IJICSA Committee; ID: CRMQ-2015-05-T-02; approved on 13 December 2016; COFEPRIS 173300410A0035/2017). The study observed the tenets of the Declaration of Helsinki. The study also adhered to the International Conference on Harmonization guideline on Good Clinical Practices, and all other applicable local regulatory requirements and laws. Before enrollment, written informed consent was obtained from all subjects, after full explanation of the nature and possible adverse events (AEs) of the study.

\subsection{Patients}

Subjects 55 years of age or older with active nAMD in one eye confirmed by fluorescein angiography (FA) were recruited. All angiographic subtypes of nAMD were enrolled. The study population comprised ranibizumab and any other anti-VEGF drug or photodynamic therapy (PDT) naïve patients. Other inclusion criteria were a best corrected visual acuity (BCVA) of 20/30 to 20/200 and absence of other ocular diseases that could affect the BCVA. Key exclusion criteria were subretinal hemorrhage of the central area of the fovea (hemorrhage $>50 \%$ of the total lesion area or 1 disc area in size), subfoveal fibrosis, or atrophy, in the study eye. Patients with retinal vein/artery occlusion, diabetic retinopathy, bilateral nAMD, study eye refractive error $>4$ diopters of myopia, angioid streaks, or precursors of $\mathrm{CNV}$ in either eye secondary to other etiologies, glaucoma and any other subjacent retinal or optic nerve condition were also excluded from the study. Additional exclusion criteria were medical history of myocardial infarction and/or stroke within 6 months before recruitment, any prior intravitreal anti-VEGF or corticosteroid injections, and any prior laser treatment and or intravitreal steroid implant such as Ozurdex $®$.

\subsection{Clinical evaluations and Study Treatment}

At baseline, all eligible participants underwent an ophthalmologic examination including BCVA measurement with early treatment diabetic retinopathy study (ETDRS) chart, fundus examination with a noncontact 90-diopter lens, slit-lamp examination, intraocular pressure (IOP) measurement using Goldmann applanation tonometry, FA (FF 450 Plus, Carl Zeiss Meditech, Inc., Dublin, CA.) and optical coherence tomography (OCT) (Cirrus 5000, Carl Zeiss Meditech, Inc., Dublin, CA.). Included subjects received in the study eye a single IVT injection of ranibizumab $0.5 \mathrm{mg}$ at baseline. Then subjects were randomly assigned to 1 of 2 treatment groups: RBZ-TALF and RBZ-pro re nata (RBZPRN). Subjects from the RBZ-TALF group were instructed to instill in the ocular surface of the study eye one drop of a liposomal formulation containing TA at $2 \mathrm{mg} / \mathrm{mL}(0.2 \%)$ four times a day for the entire follow-up (12 months). Clinical evaluation and analysis of compliance to TALF therapy was scheduled as follows: every week after the baseline visit for the first month and monthly until the end of the follow-up. Compliance of the subjects to the TA-LF therapy was evaluated through a patient care journal as follows: $A D=$ (RA)100/IA; where AD is adherence, RA corresponds to the registered applications, and IA represents the indicated number of applications. A value of adherence $<90 \%$ was considered as compliance failure, and in that case, the patient was excluded from the statistical analysis. 
On the other hand, subjects from the RBZ-PRN group received two more IVTs of ranibizumab $0.5 \mathrm{mg}$, one at the beginning of second month and the other at the beginning of the third month of the follow-up.

Retreatment with IVTs of RBZ 0.5 mg (Lucentis, Novartis, S.A. de C.V., Ciudad de Mexico, Mexico) was considered in RBZ-TALF and RBZ-PRN groups according to PrONTO study criteria [45]. Additional reinjections were administered if certain changes were observed by the evaluating physician including loss of visual acuity of at least 5 letters compared to the highest BCVA with objective evidence of fluid in the macula by OCT, an increase of at least $100 \mu \mathrm{m}$ regarding the CFT in OCT compared with the lowest $\mathrm{CFT}$, new appearance of macular hemorrhage, new area of classic CNV, or a persistent presence of fluid on OCT at least one month after the previous injection. If a retreatment (reinjection) was needed, the patient was instructed to return at the next week and then at the next scheduled visit for follow-up.

\subsection{Safety Assessment}

Safety of TALF therapy as an adjuvant to intravitreal RBZ therapy was assessed through the collection and summary of ocular and non-ocular AEs at all study visits. AEs were recorded whether informed by enrolled patients, determined by study site personnel, or other means. Vital signs measurements were done at baseline, and monthly until the end of the follow up (12 month). Patients were removed from the study in case of any evidence of low tolerability or any AEs related to the TALF topical therapy, such as ocular surface problems (ie, corneal ulcers, opacities, epithelial defects, conjunctival and/or episcleral injection) and anterior chamber inflammation (cell/flare). AEs were assigned standard codes for the event based upon the MedDRA Coding dictionary, version 18.1. Presence of AEs were evaluated every week throughout the first month and monthly for the rest of the follow-up. To identify AEs, at each visit, evaluations with a slit lamp of the anterior and posterior segments were performed, and the IOP measurement was recorded.

\subsection{Eficacy Assessment}

The mean change from baseline in BCVA, retinal central foveal thickness (CFT) and the average number of ranibizumab retreatments during the follow up period were considered as efficacy endpoints. The BCVA was evaluated with the ETDRS chart at $4 \mathrm{~m}$, whereas the central foveal thickness (CFT) was evaluated by OCT (Cirrus 5000, Carl Zeiss Meditech, Inc., Dublin, CA.). Changes in FA (FF450 plus fundus camera Carl Zeiss Meditech, Inc., Dublin, CA.) were also evaluated. Certified technicians performed the BCVA measurement, OCT and FA images acquisition at each visit.

\subsection{TALF preparation}

OPKO Health, Inc. (Guadalajara, Jalisco, Mexico) provided the TALF. As previously described, the preparation of TALF was made [46]. Briefly, production of self-forming, thermodynamically stable TALFs (QuSomes ${ }^{\circledR}$ ) was accomplished spontaneously upon adding polyethylene glycol glyceryl dimyristate (PEG-12) to an aqueous solution containing TA. Composition of TALF includes triamcinolone acetonide, 2.0mg; PEG-12 glyceryl dimyristate, $100 \mathrm{mg}$; citric acid anhydrous, $0.8 \mathrm{mg}$; ethyl alcohol, $14 \mathrm{~mL}$; kolliphor HS 15, $50 \mathrm{mg}$; sodium citrate dehydrate, $4.675 \mathrm{mg}$; benzalkonium chloride, $0.1 \mathrm{mg}$; and grade 2 purified water, Q.S.1.0 m. Final TA concentration in the resultant dispersion was $2 \mathrm{mg} / \mathrm{mL}$ $(0.2 \%)$. The preparation of TALF was performed in Good Manufacturing Practice (GMP) facilities and was carried out as previously described. Sterility of the formulation was guaranteed by mixing the excipients of the formulation at $121{ }^{\circ} \mathrm{C} 15 \mathrm{~min}$ and filtration of buffers and water containing TA through membranes of $0.22 \mathrm{~mm}$ pore size under aseptic conditions. The resulting formulation possesses $\mathrm{pH}$ of 5.8, viscosity of $70 \mathrm{cP}$ and 
osmolarity of $334 \mathrm{mOsm} / \mathrm{L}$. The dropper bottle containing TALF was preserved at room temperature.

\subsection{Data Analysis ad Statistical Methods}

Data were analyzed using the SPSS 22.0 software (IBM SPSS Statistics for Macintosh, version 22.0, IBM Corp, Armonk, NY). Quantitative variables were described using mean and standard deviation. Qualitative variables were described using frequencies and percentages. We performed a Mann-Whitney U test for the analysis of BCVA, CFT, IOP, number of reinjections. For the analysis of gender and study eye, a Fisher exact test was performed. Significance was defined as a $\mathrm{P}$ value $<0.05$.

\section{Results}

A total of 20 patients with clinical diagnosis of nAMD were enrolled. Two patients of the RBZ-TALF group met the criteria of compliance failure, therefore these were excluded from the statistical analysis. It is important to emphasize that the compliance failure was secondary to concerns about SARS CoV2 pandemic. Patients determined to suspend TALF therapy to avoid monthly clinical evaluation. Demographic and clinical characteristics of the included patients and study eyes are presented in Table 1.

Table 1. Demographic and clinical characteristics of patients and study eyes.

\begin{tabular}{|c|c|c|c|}
\hline & RBZ-PRN & RBZ-TALF & $p$ \\
\hline Age & $70.9 \pm 10.46$ & $71.5 \pm 6.65$ & 0.6795 \\
\hline Gender & & & 0.9577 \\
\hline Male (n) & 5 & 3 & \\
\hline Female (n) & 5 & 5 & \\
\hline Eye & & & 0.6353 \\
\hline $\mathrm{OD}(\mathrm{n})$ & 5 & 4 & \\
\hline OS (n) & 5 & 4 & \\
\hline Morbidity & & & 0.1675 \\
\hline Hipertension (n) & 5 & 2 & \\
\hline Diabetes Mellitus (n) & 0 & 3 & \\
\hline \multicolumn{4}{|l|}{ Ocular findings } \\
\hline Pseudophakic (n) & 2 & 4 & 0.2732 \\
\hline $\begin{array}{r}\text { Basal BCVA (ETDRS } \\
\text { letters) }\end{array}$ & $57.5 \pm 13.2$ & $56.1 \pm 14.8$ & 0.8113 \\
\hline Basal CFT $(\mu \mathrm{m})$ & $334.5 \pm 106.9$ & $399.1 \pm 75.6$ & 0.3721 \\
\hline
\end{tabular}

BCVA; Best corrected visual acuity, CFT; central foveal thickness, OD; oculus dexter (right eye), OS; oculus sinister (left eye), PRN; pro re nata, RBZ, ranibizumab, TALF; triamcinolone acetonide-loaded liposomes formulation.

In relation with preliminary safety findings, no AEs were related to TALF application during the follow-up period. Neither ocular surface abnormalities nor significant changes in IOP (Table 2 and Figure 1) or cataract progression in phakic eyes were observed. Relative to efficacy outcomes, we observed in the RBZ-TALF group a significant improvement in BCVA and CFT. The BCVA improvement was from $56.12 \pm 14.76$ at baseline to $67.1 \pm$ 13.4 letters $(\mathrm{p}<0.002)$ at the end of the follow-up (Table 2 and Figure 1). All patients improved at least 5 letters and 4 improved more than 15 letters from baseline. Four out of the 8 patients that completed the follow-up had a BCVA of 70 letters or better at month 12 of the evaluation. The CFT improved was from $369.1 \pm 80.5$ um at baseline to $271.3 \pm 82.9$ 
um $(\mathrm{p}<0.03)$ at the 12 month (Table 2 and Figure 1). Representative OCT images are presented in Figure 2. Representative images of fundus and fluorescein angiography studies are presented in Figure 3.

Table 2. Clinical outcomes after TALF administration as an adjuvant for anti-VEGF therapy in nAMD

BCVA CFT IOP

\begin{tabular}{|c|c|c|c|c|c|c|c|c|c|}
\hline \multirow[b]{2}{*}{ Patient } & \multicolumn{3}{|c|}{ (ETDRS letters) } & \multicolumn{3}{|c|}{$(\mu \mathrm{m})$} & \multicolumn{3}{|c|}{$(\mathrm{mmHg})$} \\
\hline & Baseline & $\begin{array}{c}\text { Month } \\
6\end{array}$ & Month 12 & Baseline & Month 6 & Month 12 & Baseline & $\begin{array}{c}\text { Month } \\
6\end{array}$ & $\begin{array}{c}\text { Month } \\
12\end{array}$ \\
\hline 1 & 75 & 75 & 80 & 413 & 334 & 333 & 14 & 14 & 15 \\
\hline 2 & 60 & 79 & 80 & 316 & 215 & 225 & 13 & 12 & 15 \\
\hline 3 & 68 & 76 & * & 319 & 245 & * & 13 & 16 & * \\
\hline 4 & 65 & 65 & 70 & 323 & 286 & 287 & 13 & 14 & 13 \\
\hline 5 & 39 & 40 & 57 & 452 & 223 & 211 & 14 & 12 & 13 \\
\hline 6 & 54 & 58 & 65 & 453 & 353 & 182 & 15 & 17 & 14 \\
\hline 7 & 34 & 35 & 40 & 446 & 272 & 198 & 16 & 16 & 15 \\
\hline 8 & 72 & 72 & 76 & 493 & 284 & 199 & 12 & 12 & 11 \\
\hline 9 & 48 & * & * & 320 & * & * & 14 & * & * \\
\hline 10 & 50 & 72 & 69 & 297 & 217 & 241 & 11 & 14 & 13 \\
\hline $\begin{array}{c}\text { Mean } \pm \\
\text { SD }\end{array}$ & $56.1 \pm 14.8$ & $\begin{array}{c}62.0 \pm \\
16.5\end{array}$ & $\begin{array}{c}67.1 \pm \\
13.4 \dagger\end{array}$ & $\begin{array}{c}399.1 \pm \\
75.6\end{array}$ & $\begin{array}{l}273 \pm \\
52.7 \dagger\end{array}$ & $\begin{array}{c}234.5 \pm \\
51.4 \dagger\end{array}$ & $13.5 \pm 1.6$ & $\begin{array}{c}13.9 \pm \\
1.9\end{array}$ & $\begin{array}{c}13.6 \pm \\
1.4\end{array}$ \\
\hline
\end{tabular}

BCVA; Best corrected visual acuity, CFT; Central foveal thickness, ETDRS; Early Treatment Diabetic Retinopathy Study, IOP; Intraocular pressure, SD; standard deviation, TALF; ; triamcinolone acetonide-loaded liposomes formulation. *; out of protocol (compliance failure), $t$; statistically significant from baseline.
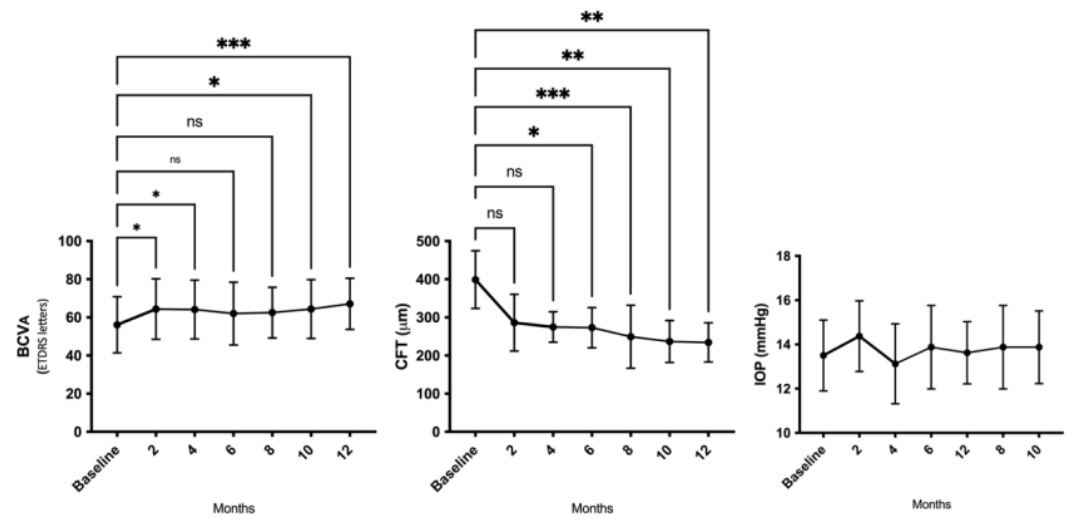

Figure 1. TALF improves BCVA and reduces CFT without IOP rising in patients with nAMD. TALF; triamcinolone acetonide-loaded liposomes formulation, nAMD; Neovascular-age related macular degeneration, BCVA; Best corrected visual acuity, ETDRS; Early Treatment Diabetic Retinopathy Study, CFT; Central foveal thickness, IOP; Intraocular pressure. ${ }^{*} ; p<0.05,{ }^{* *} ; p<0.01,{ }^{* * *} ; p<0.001$. 


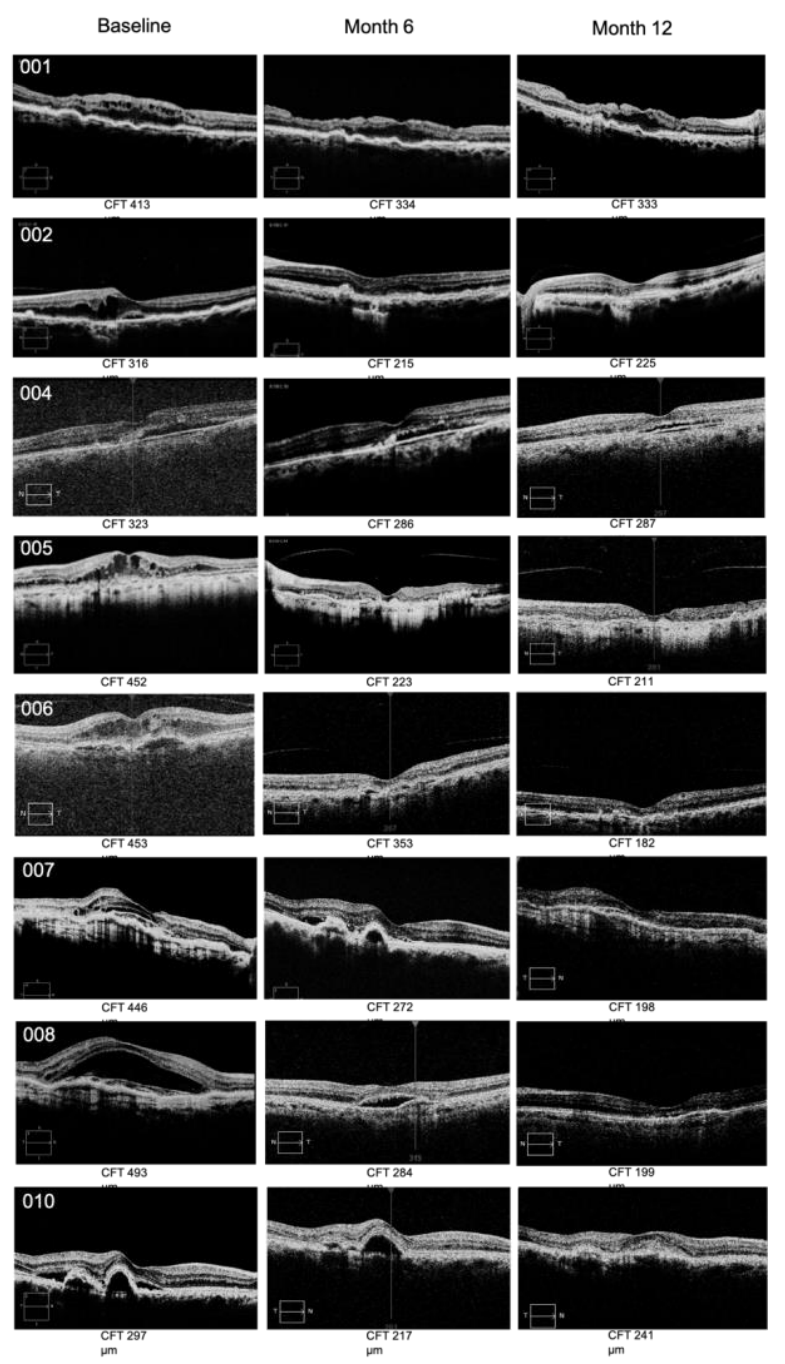

Figure 2. Representative images of CFT by OCT at baseline, month 6 and 12 of follow-up for subjects included in the RBZ-TALF group. All patients showed a consistent and progressive reduction in CFT by month 12. CFT; central foveal thickness, OCT; optical coherence tomography, RBZ; ranibizumab, TALF; triamcinolone acetonide-loaded liposomes formulation. 


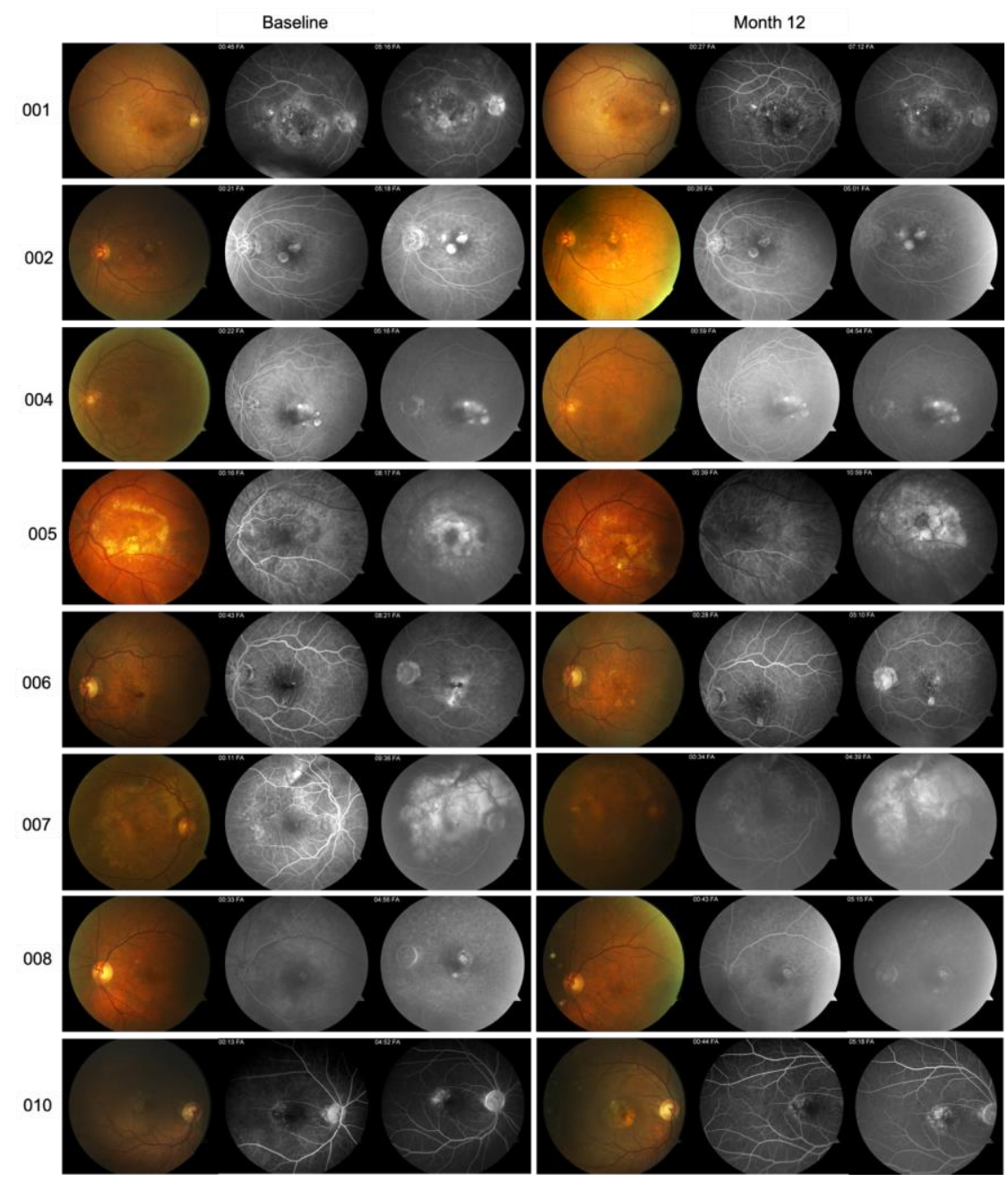

Figure 3. Representative images of fundus photograph and fluorescein angiography at baseline, and month 12 of subjects included in the RBZ-TALF group. All patients showed a decrease in the active neovascularization activity.

Lastly, when the efficacy endpoints in RBZ-TALF and RBZ-PRN groups are compared, non-significant differences are identified in BCVA or CFT (Table 3). Importantly, the average number of RBZ injections is lower in the RBZ-TALF group (Table 3). The mean injections in RBZ-TALF group were $2.5 \pm 1.4$, whereas the average number of injections in the RBZ-PRN group was $6.1 \pm 1.3$ ( $p=0004)$.

Table 3. Comparison of efficacy endpoints at the end of the follow-up between RBZ-PRN and RBZ-TALF

groups. 


\begin{tabular}{|c|c|c|c|c|c|}
\hline & \multicolumn{2}{|c|}{ RBZ -PRN } & \multicolumn{2}{|c|}{ RBZ-TALF } & \multirow{2}{*}{$\begin{array}{c}p \\
\text { Between } \\
\text { groups }\end{array}$} \\
\hline & Baseline & Month 12 & Baseline & Month 12 & \\
\hline Average BCVA (ETDRS & & & & & \\
\hline letters) & $57.5 \pm 13.2$ & $63 \pm 15.49$ & $56.1 \pm 14.8$ & $67.1 \pm 13.4$ & 0.6484 \\
\hline Gain in ETDRS letters & & $5.5 \pm 14.99$ & & $11.0 \pm 7.0$ & 0.4445 \\
\hline Average $\mathrm{CFT}(\mu \mathrm{m})$ & $334.5 \pm 106.9$ & $237.2 \pm 31.13$ & $399.1 \pm 75.6$ & $234.5 \pm 51.4$ & 0.6484 \\
\hline CFT change $(\mu \mathrm{m})$ & $97.3 \pm 95.29$ & $97.3 \pm 95.29$ & & $164.6 \pm 108.1$ & 0.1525 \\
\hline Intravitreal injections (n) & & $6.1 \pm 1.28$ & & $2.5 \pm 1.41$ & 0.0004 \\
\hline
\end{tabular}

BCVA; Best corrected visual acuity, CFT; central foveal thickness, ETDRS; Early Treatment Diabetic Retinopathy Study, PRN; pro re nata, RBZ, ranibizumab, TALF; triamcinolone acetonide-loaded liposomes formulation.

\section{Discussion}

Nowadays, intravitreal injection has become the route of preference for intraocular delivery of anti-VEGF drugs [18]. Differing from other routes, IVTs have shown the advantage of delivering higher drug concentrations into the posterior segment, specifically, into the vitreous and the retina, avoiding undesirable systemic effects. Additionally, IVTs surpass the blood-retinal barrier, which keeps most drugs out of the eye including those administered by oral and systemic routes [47]. However, there are major challenges to be overcome. For instance, the intravitreal drug administration creates a specific curve regarding its large peak concentration and a rapid decay. Literature supports the fact that the half-life of anti-VEGFs, such as RBZ, is indeed very short (half-life of 2.6 to 4.0 days) [48], making it necessary to use a higher initial dose in order to exceed therapeutic levels to allow a longer 28 day treatment interval. Moreover, mathematical modeling demonstrates that the binding activity of $0.5 \mathrm{mg}$ of RBZ is fivefold higher if given every 14 days instead of every 28 days [49]. Therefore, frequent intravitreal injections of ranibizumab are required to maintain the therapeutic effect and the visual outcomes [50, 51]. In fact, important clinical RBZ studies suggested that patients should be treated with monthly injections $[14,15,52,53]$. However, intravitreal injections are not harmless. Each intravitreal injection presents with posterior administration risk and drug class associated adverse events [54].

Furthermore, highly specialized human resources and special infrastructure are required for the administration of intravitreal injections, resulting in an expensive therapy option in developing countries [55]. Besides, IVTs itself causes discomfort to the patient, making them a potential factor of poor adherence to anti-VEGF agents treatment [56]. Therefore, intravitreal injection frequency, that negatively impacts patient health and economics, is also a major challenge to overcome [55]. For these reasons, intravitreal injections might be a burden for physicians, the health system, and patients with poor compliance $[55,56]$. Diverse therapeutic approaches, including new drugs and medical devices as well as modified therapeutic regimens like treat and extend (T\&E) have emerged in order to attend the concerns about IVTs, even, to reduce the burden of intravitreal therapy [57, 58]. Although with the PRN (pro re nata) treatment protocols and treat and extend (T\&E) regimens have been demonstrated benefits [59-63], new and safer approaches are still needed.

In this article, it was reported the feasibility and safety findings of using TALF as an adjuvant for anti-VEGF intravitreal injections. Remarkably, it was observed an important 
reduction in the number of ranibizumab intravitreal injections required to reach inactivation of nAMD and to maintain the visual outcomes that this anti-VEGF agent provides. The average number of ranibizumab retreatments was 2.5 over 12 months and all patients presented a non-active nAMD evidenced by FA study by the end of the study follow-up. This number of injections is surprisingly less when it is compared with the informed number of injections in pivotal anti-VEGF studies. For example, one of the largest studies, the MARINA trial, a randomized and double blind phase III sham-controlled trial, designed to administer monthly injections of ranibizumab and posteriorly determine its efficacy and safety in the treatment of occult and minimally-classic neovascular age-related macular degeneration, reported an improvement in BCAVA of 7.2 ETDRS letters with 13 intravitreal injection per patient in a period of 12 months [64]. Meanwhile, the PrONTO trial, a non-randomised and prospective trial designed mainly to administer intravitreal RBZ monthly for 3 months and investigate its efficacy followed by a guided clinical and OCT appearances as-required dosing regimen, reported an improvement in BCVA of 9.3 ETDRS letters with 5.5 intravitreal injection per patient in a period of 12 months [59]. In this TALF study, application of topical liposomes containing triamcinolone as an adjuvant to intravitreal RBZ, we found an improvement in BCVA of 12.8 ETDRS letters with 2.5 intravitreal reinjections per patient in a period of 12 months. Additionally, none of the recruited patient in TALF study presented AEs. Therefore, TALF has the potential to reduce treatment burden in nAMD by significantly reducing the number of retreatments. However, these preliminary findings should be confirmed in large cohort studies in order to rigorously determined the safety and efficacy of TALF formulation.

Although the results are interesting, they are expected. TA is a synthetically modified glucocorticoid mainly used for its anti-inflammatory and immunomodulatory effect against several ocular diseases. Evidence indicates that TA decreases the secretion of VEGF into human vitreous, the level of VEGF mRNA in cultured human retinal pigment epithelial cells with induced hypoxia or oxidation $[65,66]$ and inhibits angiogenesis and the leakage of retinal blood vessels $[13,67]$. Moreover, TA has also presented a direct effect in the inflammatory process, embracing the inhibition of pro-inflammatory molecules such as tumor necrosis factor-alpha (TNF- $\alpha$ ) and the pigment-derived growth factor (PEDF) [47, 68-70]. Additionally, TA have demonstrated to downregulate intercellular adhesion molecule 1 (ICAM-1) and to reduce matrix metalloproteinases (MMPs) expression on choroidal endothelial cells [71], influencing in both, angiogenesis and inflammation in nAMD.

However, intravitreal TA and related steroids may present certain adverse effects including increased intraocular pressure and cataracts formation[72-76]. TALF is a topical formulation composed of liposomes carrying TA which release the drug in vitreous and retina in small but sustained quantities [35] and presumably extend the therapeutic effect of the anti-VEGF agents.

There are several studies in the literature evaluating the impacts of combined intravitreal injection of anti-VEGF agents and triamcinolone on AMD-affected eyes overtime. For example, in a retrospective case series, Colucciello et al. treated 30 eyes of 27 patients afflicted with nAMD with a single combined intravitreal injection of bevacizumab $(1.25$ $\mathrm{mg} / 0.05 \mathrm{~mL})$ and triamcinolone $(2 \mathrm{mg} / 0.05 \mathrm{~mL})$. Their results revealed a statistically significant reduction in foveal thickness as well as in subfoveal fluid volume $(P<0.01)$ at 8 weeks after the injection [77]. The same group conducted another interventional case-series study focused on 16 eyes of 16 patients presenting $n A M D$ and previously treated with intravitreal mono-injections of bevacizumab $(1.5 \mathrm{mg})$ without significant results in the reduction in macular exudation or improvement in the visual acuity (VA) [78]. Participants of this investigation underwent a combined intravitreal injection of bevacizumab $(1.5 \mathrm{mg})$ and triamcinolone acetonide (about $20 \mathrm{mg})$. A significant improvement $(P=0.03)$ was observed in VA at 3 months after treatment. A similar prospective clinical trial by Tao and 
Jonas [43] revealed that intravitreal bevacizumab $(1.5 \mathrm{mg})$ combined with intravitreal high-dose triamcinolone $(20-25 \mathrm{mg})$ more effectively improves vision and reduces macular thickness in eyes with nAMD after unsuccessful intravitreal bevacizumab monotherapy. The findings of these studies demonstrate that triamcinolone acetonide, either at low ( $2 \mathrm{mg}$ ) or high (up to $25 \mathrm{mg}$ ) concentration can yield encouraging results in treating nAMD when it is administered intravitreally. As we mentioned previously, intravitreal injection of TA is related to adverse effects such as increased intraocular pressure and cataracts formation. These are the reason why the use of TA or other glucocorticoids in NAMD is dismissed. We demonstrated that TALF preserve the benefic effect of TA for vitreoretinal disorders but avoiding the potential adverse events, complications and burden of using intravitreal injection of large quantities of steroids. Actually, employment of TALF as an adjuvant to intravitreal injection of ranibizumab for nAMD resulted in visual outcomes comparable with the anti-VEGF PRN regimen.

\section{Conclusions}

In conclusion, TALF has the potential to reduce treatment burden on the intravitreal anti-VEGF therapy by significantly reducing the number of intravitreal retreatments. However, using TALF as an adjuvant for anti-VEGF therapy in nAMD should be studied in a larger cohort of patients, to determine efficiently its safety and effectiveness.

Author Contributions: Formal analysis, Jose Navarro-Partida, Juan Altamirano-Vallejo and Carlos Castro-Castaneda; Investigation, Luis Aceves-Franco, Jesús Gonzalez-Cortes, Sergio Hernandez Da Mota, Jose García-Aguirre, Carlos Azuara-Galindo and Juan Armendariz-Borunda; Methodology, Jose Navarro-Partida, Juan Altamirano-Vallejo and Juan Armendariz-Borunda; Project administration, Arturo Santos; Supervision, Arturo Santos; Validation, Arturo Santos; Writing - review \& editing, Carlos Castro-Castaneda.

Funding: This research received no external funding.

Institutional Review Board Statement: The study was conducted according to the guidelines of the Declaration of Helsinki and approved by the Institutional Review Board (or Ethics Committee) of IJICSA Committee (ID: CRMQ-2015-05-T-02; approved on 13 December 2016; COFEPRIS 173300410A0035/2017)

Informed Consent Statement: Informed consent was obtained from all subjects involved in the study.

Data Availability Statement: The data presented in this study are available on request from the corresponding author. The data are not publicly available due to privacy and ethical restrictions.

Acknowledgments: We want to thank to the technical staff of Centro de Retina Medica y Quirurgica S.C. due to their incredible effort in the realization of this work.

Conflicts of Interest: The authors declare no conflict of interest.

\section{References}

1. Friedman, D.S., et al., Prevalence of age-related macular degeneration in the United States. Arch ophthalmol, 2004. 122(4): p. 564-572.

2. Resnikoff, S., et al., Global data on visual impairment in the year 2002. Bulletin of the world health organization, 2004. 82: p. 844851.

3. Wong, W.L., et al., Global prevalence of age-related macular degeneration and disease burden projection for 2020 and 2040: a systematic review and meta-analysis. The Lancet Global Health, 2014. 2(2): p. e106-e116.

4. Bressler, N.M., Age-Related Macular Degeneration Is the Leading Cause of Blindness. Jama, 2004. 291(15): p. $1900-1901$.

5. Jonas, J.B., C.M.G. Cheung, and S. Panda-Jonas, Updates on the epidemiology of age-related macular degeneration. The Asia-Pacific Journal of Ophthalmology, 2017. 6(6): p. 493-497.

6. Tsilimbaris, M.K., et al., Epidemiological and clinical baseline characteristics as predictive biomarkers of response to anti-VEGF treatment in patients with neovascular AMD. Journal of ophthalmology, 2016. 2016.

7. Tsutsumi-Miyahara, C., et al., The relative contributions of each subset of ocular infiltrated cells in experimental choroidal neovascularisation. British journal of ophthalmology, 2004. 88(9): p. 1217-1222.

8. Grossniklaus, H.E., et al., Macrophage and retinal pigment epithelium expression of angiogenic cytokines in choroidal neovascularization. Mol Vis, 2002. 8(8): p. 119-126. 
9. Grossniklaus, H.E. and W.R. Green, Choroidal neovascularization. American journal of ophthalmology, 2004. 137(3): p. 496-503.

10. Espinosa-Heidmann, D.G., et al., Quantitative enumeration of vascular smooth muscle cells and endothelial cells derived from bone marrow precursors in experimental choroidal neovascularization. Experimental eye research, 2005. 80(3): p. 369-378.

11. Killingsworth, M.C., Angiogenesis in early choroidal neovascularization secondary to age-related macular degeneration. Graefe's archive for clinical and experimental ophthalmology, 1995. 233(6): p. 313-323.

12. Sheridan, C.M., et al., The presence of AC133-positive cells suggests a possible role of endothelial progenitor cells in the formation of choroidal neovascularization. Investigative ophthalmology \& visual science, 2006. 47(4): p. 1642-1645.

13. Ebrahem, Q., et al., Triamcinolone acetonide inhibits IL-6-and VEGF-Induced angiogenesis downstream of the IL-6 and VEGF receptors. Investigative ophthalmology \& visual science, 2006. 47(11): p. 4935-4941.

14. Brown, D.M., et al., Ranibizumab versus verteporfin for neovascular age-related macular degeneration. New England Journal of Medicine, 2006. 355(14): p. 1432-1444.

15. Rosenfeld, P.J., et al., Ranibizumab for neovascular age-related macular degeneration. New England Journal of Medicine, 2006 355(14): p. 1419-1431.

16. Regillo, C.D., et al., Randomized, double-masked, sham-controlled trial of ranibizumab for neovascular age-related macular degeneration: PIER Study year 1. American journal of ophthalmology, 2008. 145(2): p. 239-248. e5.

17. Mitchell, P., et al., Ranibizumab (Lucentis) in neovascular age-related macular degeneration: evidence from clinical trials. British Journal of Ophthalmology, 2010. 94(1): p. 2-13.

18. Grzybowski, A., et al., 2018 update on intravitreal injections: Euretina expert consensus recommendations. Ophthalmologica, 2018 239(4): p. 181-193.

19. Meyer, C.H., et al., Incidence of rhegmatogenous retinal detachments after intravitreal antivascular endothelial factor injections. Acta ophthalmologica, 2011. 89(1): p. 70-75.

20. Lyall, D.A., et al., Post-intravitreal anti-VEGF endophthalmitis in the United Kingdom: incidence, features, risk factors, and outcomes. Eye, 2012. 26(12): p. 1517-1526.

21. Munk, M.R., et al., Macular atrophy in patients with long-term anti-VEGF treatment for neovascular age-related macular degeneration. Acta ophthalmologica, 2016. 94(8): p. e757-e764.

22. Abdelfattah, N.S., et al., Progression of macular atrophy in patients with neovascular age-related macular degeneration undergoing antivascular endothelial growth factor therapy. Retina, 2016. 36(10): p. 1843-1850.

23. Dugel, P.U., et al., HAWK and HARRIER: phase 3, multicenter, randomized, double-masked trials of brolucizumab for neovascular agerelated macular degeneration. Ophthalmology, 2020. 127(1): p. 72-84.

24. Pacella, E., et al., Preliminary results of an intravitreal dexamethasone implant (Ozurdex $\left.{ }^{\circledR}\right)$ in patients with persistent diabetic macular edema. Clinical Ophthalmology (Auckland, NZ), 2013. 7: p. 1423.

25. Pearson, P.A., et al., Fluocinolone acetonide intravitreal implant for diabetic macular edema: a 3-year multicenter, randomized, controlled clinical trial. Ophthalmology, 2011. 118(8): p. 1580-1587.

26. Campochiaro, P.A., et al., The port delivery system with ranibizumab for neovascular age-related macular degeneration: results from the randomized phase 2 ladder clinical trial. Ophthalmology, 2019. 126(8): p. 1141-1154.

27. Laganovska, G., First-in-human results of a refillable drug delivery implant providing release of ranibizumab in patients with neovascular $A M D$. American Academy of Ophthalmology, Retina Subspecialty Day, 2012.

28. Humayun, M., et al., Implantable micropump for drug delivery in patients with diabetic macular edema. Translational vision science \& technology, 2014. 3(6): p. 5-5.

29. Bisht, R., et al., Nanocarrier mediated retinal drug delivery: overcoming ocular barriers to treat posterior eye diseases. Wiley Interdisciplinary Reviews: Nanomedicine and Nanobiotechnology, 2018. 10(2): p. e1473.

30. Gaudana, R., et al., Recent perspectives in ocular drug delivery. Pharmaceutical research, 2009. 26(5): p. 1197-1216.

31. F Fangueiro, J., et al., Ocular drug delivery-new strategies for targeting anterior and posterior segments of the eye. Current pharmaceutical design, 2016. 22(9): p. 1135-1146.

32. Bravo-Osuna, I., et al., Pharmaceutical microscale and nanoscale approaches for efficient treatment of ocular diseases. Drug delivery and translational research, 2016. 6(6): p. 686-707.

33. Kumar, R., Lipid-based nanoparticles for drug-delivery systems, in Nanocarriers for Drug Delivery. 2019, Elsevier. p. 249-284.

34. Mishra, G.P., et al., Recent applications of liposomes in ophthalmic drug delivery. Journal of drug delivery, 2011. 2011.

35. Altamirano-Vallejo, J.C., et al., Characterization and pharmacokinetics of triamcinolone acetonide-loaded liposomes topical formulations for vitreoretinal drug delivery. Journal of Ocular Pharmacology and Therapeutics, 2018. 34(5): p. 416-425.

36. Gonzalez-De la Rosa, A., et al., Novel triamcinolone acetonide-loaded liposomes topical formulation for the treatment of cystoid macular edema after cataract surgery: A pilot study. Journal of Ocular Pharmacology and Therapeutics, 2019. 35(2): p. 106-115.

37. Navarro-Partida, J., et al., Safety and Tolerability of Topical Ophthalmic Triamcinolone Acetonide-Loaded Liposomes Formulation and Evaluation of Its Biologic Activity in Patients with Diabetic Macular Edema. Pharmaceutics, 2021. 13(3): p. 322.

38. Navarro-Partida, J., et al., Topical Triamcinolone Acetonide-Loaded Liposomes as Primary Therapy for Macular Edema Secondary to Branch Retinal Vein Occlusion: A Pilot Study. Journal of Ocular Pharmacology and Therapeutics, 2020. 36(6): p. 393-403.

39. Gopal, L. and T. Sharma, Use of intravitreal injection of triamcinolone acetonide in the treatment of age-related macular degeneration. Indian journal of ophthalmology, 2007. 55(6): p. 431.

40. Jonas, J.B., et al., Duration of the effect of intravitreal triamcinolone acetonide in eyes with exudative age-related macular degeneration Journal of Ocular Pharmacology \& Therapeutics, 2006. 22(3): p. 194-199. 
41. Ahmadieh, H., et al., Intravitreal bevacizumab versus combined intravitreal bevacizumab and triamcinolone for neovascular age-related macular degeneration: six-month results of a randomized clinical trial. Retina, 2011. 31(9): p. 1819-1826.

42. El Matri, L., et al., Intravitreal injection of triamcinolone combined with bevacizumab for choroidal neovascularization associated with large retinal pigment epithelial detachment in age-related macular degeneration. Graefe's Archive for Clinical and Experimental Ophthalmology, 2010. 248(6): p. 779-784.

43. Tao, Y. and J.B. Jonas, Intravitreal bevacizumab combined with intravitreal triamcinolone for therapy-resistant exudative age-related macular degeneration. Journal of ocular pharmacology and therapeutics, 2010. 26(2): p. 207-212.

44. Riazi-Esfahani, M., et al., Intravitreal bevacizumab versus combined bevacizumab and triamcinolone acetonide for neovascular age-related macular degeneration. Journal of ophthalmic \& vision research, 2008. 3(2): p. 95.

45. Lalwani, G.A., et al., A variable-dosing regimen with intravitreal ranibizumab for neovascular age-related macular degeneration: year 2 of the PrONTO Study. American journal of ophthalmology, 2009. 148(1): p. 43-58. e1.

46. Altamirano-Vallejo, J.C., et al., Characterization and Pharmacokinetics of Triamcinolone Acetonide-Loaded Liposomes Topical Formulations for Vitreoretinal Drug Delivery. J Ocul Pharmacol Ther, 2018. 34(5): p. 416-425.

47. Umland, S.P., et al., The inhibitory effects of topically active glucocorticoids on IL-4, IL-5, and interferon- $\gamma$ production by cultured primary CD4+ T cells. Journal of allergy and clinical immunology, 1997. 100(4): p. 511-519.

48. Gaudreault, J., et al., Preclinical pharmacokinetics of Ranibizumab (rhuFabV2) after a single intravitreal administration. Investigative ophthalmology \& visual science, 2005. 46(2): p. 726-733.

49. Stewart, M.W., et al., Pharmacokinetic rationale for dosing every 2 weeks versus 4 weeks with intravitreal ranibizumab, bevacizumab, and aflibercept (vascular endothelial growth factor Trap-eye). Retina, 2012. 32(3): p. 434-457.

50. Martin, D.F., et al., Ranibizumab and bevacizumab for treatment of neovascular age-related macular degeneration: two-year results. Ophthalmology, 2012. 119(7): p. 1388-1398.

51. Chakravarthy, U., et al., Alternative treatments to inhibit VEGF in age-related choroidal neovascularisation: 2-year findings of the IVAN randomised controlled trial. The Lancet, 2013. 382(9900): p. 1258-1267.

52. Chen, Y. and F. Han, Profile of ranibizumab: efficacy and safety for the treatment of wet age-related macular degeneration. Therapeutics and clinical risk management, 2012. 8: p. 343.

53. Heier, J.S., et al., Intravitreal aflibercept (VEGF trap-eye) in wet age-related macular degeneration. Ophthalmology, 2012. 119(12): $\mathrm{p}$ 2537-2548.

54. Falavarjani, K.G. and Q. Nguyen, Adverse events and complications associated with intravitreal injection of anti-VEGF agents: a review of literature. Eye, 2013. 27(7): p. 787-794.

55. Azad, R., P. Chandra, and R. Gupta, The economic implications of the use of anti-vascular endothelial growth factor drugs in age-related macular degeneration. Indian journal of ophthalmology, 2007. 55(6): p. 441

56. Droege, K.M., et al., Adherence to ranibizumab treatment for neovascular age-related macular degeneration in real life. Graefe's Archive for Clinical and Experimental Ophthalmology, 2013. 251(5): p. 1281-1284.

57. Barthelmes, D., et al., Two year outcomes of "treat and extend" intravitreal therapy using aflibercept preferentially for neovascular agerelated macular degeneration. Retina, 2018. 38(1): p. 20-28.

58. Gillies, M.C., et al., Long-term outcomes of treatment of neovascular age-related macular degeneration: data from an observational study. Ophthalmology, 2015. 122(9): p. 1837-1845.

59. Fung, A.E., et al., An optical coherence tomography-guided, variable dosing regimen with intravitreal ranibizumab (Lucentis) for neovascular age-related macular degeneration. American journal of ophthalmology, 2007. 143(4): p. 566-583. e2.

60. Johnston, R.L., et al., A retrospective study of ranibizumab treatment regimens for neovascular age-related macular degeneration (nAMD) in Australia and the United Kingdom. Advances in therapy, 2017. 34(3): p. 703-712.

61. Holz, F.G., et al., Safety and efficacy of a flexible dosing regimen of ranibizumab in neovascular age-related macular degeneration: the SUSTAIN study. Ophthalmology, 2011. 118(4): p. 663-671.

62. Wykoff, C.C., et al., Prospective trial of treat-and-extend versus monthly dosing for neovascular age-related macular degeneration: TREXAMD 1-year results. Ophthalmology, 2015. 122(12): p. 2514-2522.

63. Brand, C. and S. Lacey, One-year outcomes with ranibizumab in treatment naïve patients with neovascular age-related macular degeneration: an interim analysis from the LUMINOUS ${ }^{\mathrm{TM}}$ study. Investigative Ophthalmology \& Visual Science, 2016. 57(12): p. 515-515.

64. Rosenfeld, P., et al., MARINA Study Group Ranibizumab for age-related macular degeneration. N Engl J Med, 2006. 355(14): p. 14191431.

65. Brooks, H.L., et al., Vitreous Levels of Vascular Endothelial Growth Factor and Stromal-DerivedFactor 1 in Patients With Diabetic Retinopathy and Cystoid Macular Edema Beforeand After Intraocular Injection of Triamcinolone. Archives of ophthalmology, 2004. 122(12): p. $1801-1807$.

66. Matsuda, S., et al., Vascular endothelial growth factor reduced and connective tissue growth factor induced by triamcinolone in ARPE19 cells under oxidative stress. Investigative ophthalmology \& visual science, 2005. 46(3): p. 1062-1068.

67. Edelman, J.L., D. Lutz, and M.R. Castro, Corticosteroids inhibit VEGF-induced vascular leakage in a rabbit model of blood-retinal and blood-aqueous barrier breakdown. Experimental eye research, 2005. 80(2): p. 249-258.

68. Obata, R., et al., Triamcinolone acetonide suppresses early proangiogenic response in retinal pigment epithelial cells after photodynamic therapy in vitro. British journal of ophthalmology, 2007. 91(1): p. 100-104.

69. Floman, N. and U. Zor, Mechanism of steroid action in ocular inflammation: Inhibition of prostaglandin production. Investigative ophthalmology \& visual science, 1977. 16(1): p. 69-73. 
70. Kang, B.-S., et al., Inhibitory effects of anti-inflammatory drugs on interleukin-6 bioactivity. Biological and Pharmaceutical Bulletin, 2001. 24(6): p. 701-703.

71. Sarao, V., et al., Intravitreal steroids for the treatment of retinal diseases. The Scientific World Journal, 2014. 2014

72. Arikan, G., A. Osman Saatci, and F. Hakan Oner, Immediate intraocular pressure rise after intravitreal injection of ranibizumab and two doses of triamcinolone acetonide. Int J Ophthalmol, 2011. 4(4): p. 402-5.

73. Chan, C.K., et al., Ocular-hypertensive response and corneal endothelial changes after intravitreal triamcinolone injections in Chinese subjects: a 6-month follow-up study. Eye (Lond), 2005. 19(6): p. 625-30.

74. Veritti, D., et al., Drug safety evaluation of intravitreal triamcinolone acetonide. Expert Opin Drug Saf, 2012. 11(2): p. 331-40.

75. Chu, Y.K., et al., Objective evaluation of cataract progression associated with a high dose intravitreal triamcinolone injection. Eye (Lond), 2008. 22(7): p. 895-9.

76. Smithen, L.M., et al., Intravitreal triamcinolone acetonide and intraocular pressure. Am J Ophthalmol, 2004. 138(5): p. 740-3.

77. Colucciello, M., Intravitreal bevacizumab and triamcinolone acetonide combination therapy for exudative neovascular age-related macular degeneration: short-term optical coherence tomography results. Journal of ocular pharmacology and therapeutics, 2008. 24(1): p. 1524.

78. Jonas, J.B., et al., Combined intravitreal bevacizumab and triamcinolone in exudative age-related macular degeneration. Acta ophthalmologica, 2010. 88(6): p. 630-634 